\title{
Metastatic Malignant Mesothelioma Presenting With Cardiogenic Shock
}

\author{
Obsinet Merid $^{\text {a }}$, Tesfaye A. Telila ${ }^{\text {a }}$, Robel Haile ${ }^{a}$, Mingchen Song ${ }^{\text {a, b }}$
}

\begin{abstract}
Metastatic tumor progression to the pericardium is generally characterized by pericardial effusion with or without tamponade. It is extremely rare for tumor to metastasize to the pericardium and cause constrictive pericarditis and tamponade physiology. We report a case of metastatic malignant mesothelioma admitted to our facility for acute hypoxic respiratory failure and cardiogenic shock due to extensive pericardial metastasis and trapped heart. This was confirmed during post-mortem evaluation, which showed malignant mesothelioma involving the anterior mediastinum and partially encasing the heart and the root of the aorta in addition to fibrous pericarditis and pericardial adhesion.
\end{abstract}

Keywords: Malignant mesothelioma; Cardiogenic shock

\section{Introduction}

Metastasis to the heart is not uncommon, but primary cardiac tumors are rare $(0.01 \%$ and $0.1 \%$ on post-mortem studies) [1]. The incidence of cardiac metastases has increased over the last 30 years, which is attributed to increase in life expectancy and advances in cancer diagnosis and treatment [2].

Clinical presentations of cardiac metastases are extremely variable. It has been reported that cardiac metastasis may be the first or even the only manifestation of an undiagnosed malignant neoplasm, usually diagnosed during post-mortem investigations.

We present a 73-year-old Caucasian gentleman with a new diagnosis of malignant mesothelioma who presented with progressive dyspnea and cardiogenic shock due to extensive pericardial and aortic root metastasis.

Manuscript accepted for publication January 11, 2017

aDepartment of Internal Medicine, Southern Illinois University School of Medicine, Springfield, IL, USA

${ }^{b}$ Corresponding Author: Mingchen Song, Department of Internal Medicine, Southern Illinois University School of Medicine, Springfield, IL 62794, USA. Email: msong@siumed.edu

doi: https://doi.org/10.14740/jmc2739w

\section{Case Report}

The patient was a 73-year-old Caucasian male with a history of coronary artery disease, hyperlipidemia, syncope, former cigarette smoker, remote asbestos exposure and recent diagnosis of atrial fibrillation who had multiple admissions for evaluation of progressive dyspnea. He was initially hospitalized for a workup of exertional dyspnea and severe exercise intolerance associated with chest pain and new onset atrial fibrillation. Initial computed tomography (CT) of the chest revealed a significant pleural thickening (Fig. 1), for which he was scheduled for a video-assisted thoracoscopic pleural biopsy for suspected mesothelioma. However, he was suddenly decompensated and transferred to intensive care unit (ICU) for acute hypoxic respiratory failure and profound hypotension of unclear etiology.

Physical examinations revealed a thin built Caucasian male in severe respiratory distress, raised JVP, diminished heart sounds, bibasilar crackles and trace lower extremity pitting edema. Repeat chest X-ray showed acute pulmonary edema with left-sided pleural thickening as seen on admission films. Because of his worsening respiratory status, he initially tried on non-invasive positive pressure ventilation which he failed, requiring invasive mechanical ventilation. In addition, he remained hypotensive despite aggressive fluid resuscitation, later requiring multiple vasopressors. Electrocardiography showed sinus rhythm with sinus arrhythmia with first

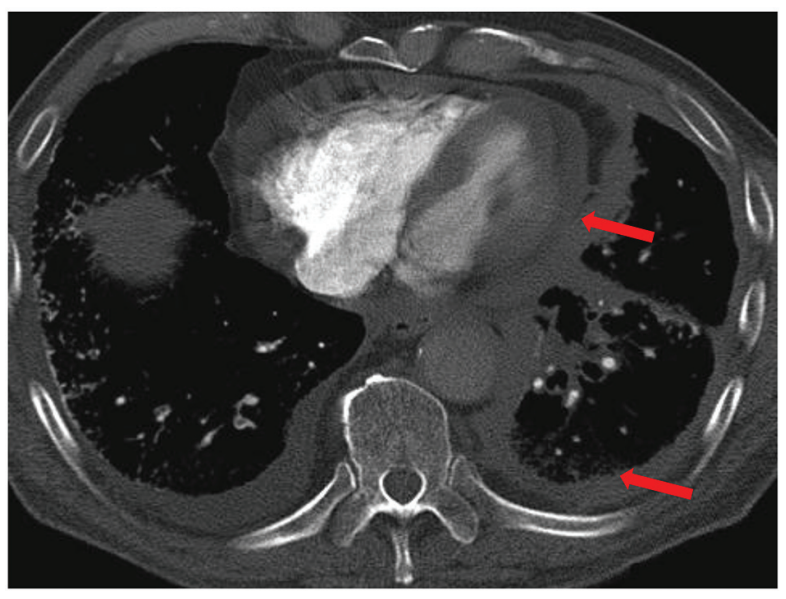

Figure 1. CT of chest showed significant pleural thickening (arrow). 


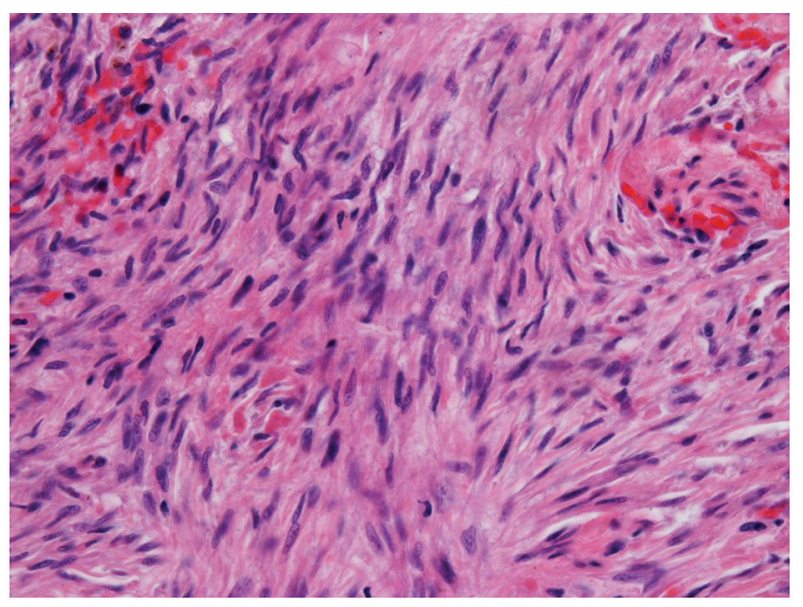

Figure 2. Sarcomatoid type of malignant mesothelioma.

degree AV block, low voltage QRS and non-specific T wave abnormalities.

Given his profound hypotension and respiratory failure, while awaiting further workup for an alternative explanation, he was started on treatment for presumed septic shock secondary to healthcare-associated pneumonia. Transthoracic echocardiogram showed small left ventricle with normal wall thickness, normal global systolic function and impaired relaxation. There were no significant valve lesions or pericardial effusion.

Despite high-dose vasopressors and aggressive hemodynamic support, the patient continued to be hypotensive with systolic blood pressure ranging from 30 to $70 \mathrm{~mm} \mathrm{Hg}$. Culture results from all potential foci of infection were all unrevealing. Subsequently, patient developed signs of end organ hypo-perfusion and multi-organ failure, which prompted a family discussion regarding the goals of care and prognosis. The family then opted out for comfort measures only. Later, all life supports were discontinued and patient expired shortly after extubation.

Post-mortem examination demonstrated sarcomatoid type of malignant mesothelioma (Fig. 2) entirely invading the left lung, part of the right lung, aortic root and most of the left ventricular wall (Fig. 3). The epicardial and pericardial surfaces were shaggy and did show fibrous pericarditis and extensive pericardial adhesions. It is now retrospectively evident that involvement of the pericardium, left ventricular wall and aortic root all contributed to the development of cardiogenic shock.

\section{Discussion}

Metastasis to the heart and pericardium is commonly from breast cancer, lung cancer, melanoma, leukemia, and lymphoma. The frequency of metastatic tumors to the pericardium, myocardium, great vessels, or coronary arteries is between $0.7 \%$ and $3.5 \%$ on post-mortem studies and up to $9.1 \%$ in patients with known malignancies [1].

The major pathways through which tumors might involve the heart and pericardium include retrograde lymphatic extension, direct contiguous extension, or hematogenous [1]. Car-

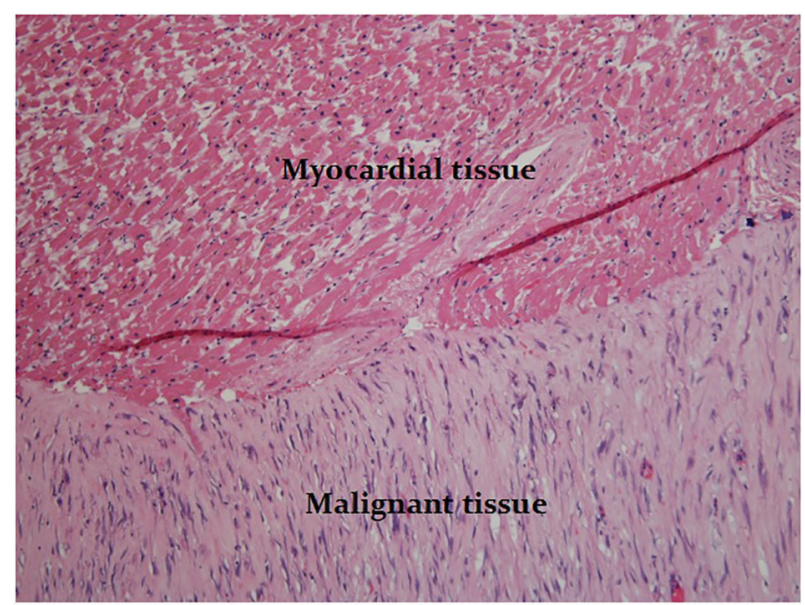

Figure 3. Tumor cells invaded the left ventricular wall.

diac metastasis typically has poor prognosis.

The most common site for cardiac metastasis is pericardium, comprising 64-69\% of all cardiac metastases [3]. Tumor metastasis to the pericardium may initially present as pericarditis with or without formation of pericardial effusion. Depending on their size and rate of accumulation, malignant pericardial effusions may be symptomatic or silent. Less commonly, as in our patient, deposits of pericardial and aortic root metastases may also compromise cardiac output via a combination of constrictive and tamponade physiology. The other common sites for cardiac metastasis are epicardium (25-34\%) and myocardium (29-32\%) [3].

The clinical manifestations of cardiac metastases are nonspecific and depend on their location. Most cardiac metastases are clinically silent and most are diagnosed only during postmortem evaluation as in our case. Clinical manifestations of cardiac metastases are difficult to distinguish from other causes of cardiopulmonary diseases. As in our case, local extensions to the heart can clinically manifest with progressive severe exercise intolerance and new onset atrial fibrillation. However, the development of cardiogenic shock due to pleuro-pericardial metastasis and constrictive physiology and a trapped heart is a less common manifestation [4].

Diagnosis of cardiac metastasis may be difficult but should be suspected in patients with known or suspected malignancy presenting with new and otherwise unexplained cardiac symptoms [4].

Physical examination may reveal distant heart sounds suggesting a malignant pericardial effusion, JVD, new murmurs due to intracardiac masses or a pericardial friction rub from pericarditis. ECG is usually non-specific. The most common abnormalities are non-specific ST-T wave changes and new atrial arrhythmias. Low voltage and electric alternant may indicate the presence of a pericardial effusion $[1,4]$.

Imaging studies are essential for the diagnosis of cardiac metastasis. Chest X-ray may demonstrate cardiomegaly from a pericardial effusion. Echocardiography is the initial imaging modality to detect pericardial effusions and provide information on the location, size, and mobility of cardiac masses $[1,4]$.

Cardiac magnetic resonance imaging (MRI), computed 
tomography (CT), and positron emission tomography can provide non-invasive characterization of cardiac metastasis and extent of soft tissue involvement. Cardiac CT and MRI are also useful for identifying direct extension of tumor from the mediastinum [4].

Histology remains the most definitive method for diagnosis. Malignant cells can be identified in the majority of malignant pericardial effusions drained by pericardiocentesis, and the cytology of malignant cells has an extraordinarily high correlation with the histological diagnosis. Other diagnostic tools include endomyocardial biopsy and open biopsy may be sometimes indicated [1].

The management of cardiac metastases depends on the clinical presentation. Cardiac tamponade requires immediate pericardiocentesis. Metastasis-induced arrhythmias can sometimes be transiently managed by the use of antiarrhythmic medications or by radiofrequency ablation [4]. Surgical resection is generally reserved for patient with good functional status and when technically feasible. Radiotherapy and chemotherapy can also be useful tools for the treatment of certain cardiac metastases [4]. The most important goals of intervention should include palliation of symptoms and prevention or delay of symptom recurrence [4].

\section{Conclusion}

Cardiac metastases are often clinically silent, and they should always be considered in any individual with new cardiac symptoms and known malignancy or risk factors.

The clinical presentations of cardiac metastases are nonspecific and vary based on the anatomic localization of tumor involvement. Echocardiography is the initial imaging test of choice for the detection of cardiac metastasis. Cardiac MRI, cardiac CT, and positron emission tomography are helpful to characterize and identify the extent of both cardiac and extracardiac diseases.

Treatment of cardiac metastases depends on their immediate cardiac complications, the clinical context, prognosis, and functional status of the patient. Cardiogenic shock secondary to either primary pleuro-pericardial or metastatic mesothelioma is a rare entity and diagnosis requires a high index of clinical suspicion.

\section{Financial Disclosure}

None.

\section{Conflicts of Interest}

None.

\section{References}

1. Bussani R, De-Giorgio F, Abbate A, Silvestri F. Cardiac metastases. J Clin Pathol. 2007;60(1):27-34.

2. Wenger NK. Pericardial disease in the elderly. Cardiovasc Clin. 1992;22(2):97-103.

3. Butany J, Leong SW, Carmichael K, Komeda M. A 30year analysis of cardiac neoplasms at autopsy. Can J Cardiol. 2005;21(8):675-680.

4. Goldberg AD, Blankstein R, Padera RF. Tumors metastatic to the heart. Circulation. 2013;128(16):1790-1794. 